\title{
Fall Incidence and the Use of Psychotropic, Opioid or Cardiovascular Medications
}

\author{
Iniguez R01, Trueblood PR ${ }^{1 *}$, Tracz $\mathrm{S}^{2}$ and Zarrinkhameh $\mathrm{L}^{1}$ \\ ${ }^{1}$ Department of Physical Therapy, California State University, USA \\ ${ }^{2}$ Kremen School of Education and Human Development, California State University, \\ USA
}

\section{Research Article \\ Volume 1 Issue 1}

Received Date: September 15, 2018

Published Date: October 22, 2018

DOI: $10.23880 /$ aphot- 16000105

*Corresponding author: Peggy R Trueblood, California State University, Fresno, 5315 Campus Drive M/S PT29 Fresno, CA 93740, USA, Tel: (559) 278-3008; Fax: (559)278-3635; Email: peggyt@csufresno.edu

\section{Abstract}

Background: Medications are one identifiable risk factor for falls. In addition to polypharmacy, certain medications have been associated with an increased risk for falls. The purpose of this study is to assess if the most frequently prescribed medication categories: psychotropic, opioid and cardiovascular are associated with an increased incidence of falls in community dwelling adults 65 years and older.

Methods: This retrospective observational study used data collected from subjects who participated in the Senior Awareness and Fall Education Balance Screening Program throughout Fresno County from 2007-2014. A review was completed by one person to identify psychotropic, opioid, and cardiovascular medications. Due to the abundance of cardiovascular medications, this group was classified into 12 subcategories. Participants were identified as a faller if they had experienced $\geq 2$ falls in the last year and $\leq 1$ fall served as the comparison group.

Results: A total of 509 participants were included. A stepwise logistic regression analysis was used to examine the predictor of falls. The overall model was statistically significant $(\mathrm{P}<.001)$. Furthermore, $70.3 \%$ of fallers and non-fallers were categorized correctly when subjects were taking psychotropic and opioid medications. Chi-square test analysis detected a statistically significant association between reported incidence of falls and use of either psychotropic or opioid but not cardiovascular medication category. When cardiovascular drug classes were subcategorized, the use of antiarrhythmic medication was statically significant. The strongest predictor for falls, however, was use of psychotropic and opioid medications combined. Subjects taking at least one medication from psychotropic drug class were 2.4 times (OR 2.4, CI 1.56-3.64) more likely to have reported a fall. Those taking opioid medications were only 2.1 times (OR 2.1, CI 1.17-2.23) more likely to have reported a fall. But, if the subjects were taking medications from both psychotropic and opioid classifications, they were 4.3 times (OR 4.3, CI 1.96-9.64) more likely to report a fall. 


\section{Annals of Physiotherapy \& Occupational Therapy}

Conclusion: Identification of older adults who consume anti-arrhythmic, opioids, or psychotropic medications could allow for patient education on adverse drug reactions, early intervention of fall prevention, or referral to physician/pharmacist for medication consolidation, in line with new national initiative for decreased opioid use.

Keywords: Fall incidence; Medication; Community-dwelling older adults; Polypharmacy

Abbreviations: CNS: Central Nervous System; OR: Odds Ratio; ACE: Angiotensin Converting Enzyme; CCB: Calcium Channel Blocker; ARB: Angiotensin Receptor Blocker.

\section{Introduction}

The fastest growing population segment in the United States consist of adults aged 65 years and older [1]. They represent $13 \%$ of the population, which is approximately 40.3 million individuals [1]. From this population segment, one-third of community-dwelling adults experience a fall each year [2]. The etiology of falls is multi factorial $[2,3]$. Medications are one identifiable risk factor $[4,5]$. Older adults, in the United States, consume $33 \%$ of all prescription medications for management of multiple comorbidities [6]. Various studies have identified polypharmacy, the use of four or more medications, as a contributing factor to increased fall risk [7-10]. Psychotropic, opioid, and cardiovascular medications are the medications most frequently prescribed [11]. There are limited studies that assess whether an association exists between the most commonly prescribed medications in older adults and the incidence of falls [1214]. For this reason, this study seeks to evaluate the relationship between fall incidence in community dwelling adults aged 65 years and older who consume psychotropic, opioid, or cardiovascular medications. Psychotropic medications are used in the treatment of certain disorders, such as depression and anxiety [15]. Psychotropic medications are agents that cross the blood brain barrier and primarily affect the central nervous system (CNS) [16]. Medications in this pharmaceutical category have the potential to produce adverse drug reactions [17]. The association between psychotropic medications and the incidence of falls is well documented within the literature [18-21]. In a meta-analysis, Leipzig, et al. [18] reported an increased fall incidence with use of any medication classified as a psychotropic drug. An estimated odds ratio (OR) of 1.73 (95\% CI, 1.52-1.97; $\mathrm{P}<.001$ ) for individuals 60 years of age and older was identified [18]. This meta-analysis included studies involving participants in nursing homes and community settings. This heightened incidence of falls is a consistent finding, even when psychotropic medications are classified based on pharmacological subclasses [18-20]. For example, benzodiazepines and antidepressants are associated with the incidence of falls with an OR of 1.48 (95\% CI, 1.230166) and 1.66 (95\% CI, 1.40-1.95), respectively [18]. Similar associations are reported in studies that include community-dwelling adults aged 65 years and older [20-22].

In recent years much attention has been given to the concept of pain management in adults 65 years and older [23]. Opioid medications are commonly prescribed for non-cancer related chronic pain in odder adults. For example, patients with moderate to severe osteoarthritis, who did not benefit from less potent pain medication, are often prescribed opioid medication [24-25]. This type of analgesic medication has an inhibitory action on pain signals. The use of opioid medications and their association with fall incidence in older adults has been assessed throughout the literature [23-27]. However, no common consensus has been formulated.

In addition, to the use of opioid medications, cardiovascular medications are commonly prescribed to older adults for the treatment of diseases related to the structure and function of the heart and blood vessels [16]. All classes of cardiovascular medications have the potential to produce adverse drug reactions that may include bradycardia, orthostatic hypotension, or lightheadedness; all of which can potentially precipitate the occurrence of a fall [16]. The quality and quantity of research assessing the relationship between cardiovascular medications and fall incidence in community-dwelling adults 65 years or older is limited $[14,18,26,28-30]$. Due to the abundance of cardiovascular pharmacological classes, sub categorization of these agents varies between studies [13,29-30]. In a metaanalysis, Leipzig, et al. [27] reported that certain cardiovascular medications (e.g., type 1a anti-arrhythmic, cardiac glycoside, and diuretics) have a statistically significant association $(\mathrm{P}<.05)$ with incidence of falls. The consumption of either one of these medications has 
resulted in a reported OR of 1.59 (95\% CI, 1.02-2.48), 1.22 (95\% CI, 0.92-1.58) respectively [27]. In another metaanalysis, Zang [29] found that cardiovascular medications, used for the treatment of hypertension, are not associated with heightened incidence falls. A change in cardiovascular medication is associated with an increased risk of hospital admission due to injury, such as a fall/or fracture with an OR 1.54 (95\% CI, 1.17-2.03, P=.002) [29].

Although several studies have alluded to an association between fall incidence and the use of psychotropic, opioid, or cardiovascular medications, doctors continue to prescribe these potentially inappropriate medications to community-dwelling adults 65 years and older. Therefore, the aim of this study is to compare fallers to non-fallers who are consuming psychotropic, opioid, or cardiovascular medications. The hypothesis questions whether community-dwelling adults 65 years of age and older who consume psychotropic, opioid, or cardiovascular medications will have an association with fall incidence. The null hypothesis states there is no association between the use of psychotropic, opioid, or cardiovascular medication and fall incidence.

\section{Methods}

This retrospective study used data collected from the Senior Awareness and Fall Education Balance Screening Program held throughout Fresno County from 2007-2014. Only the information collected during the screenings that is pertinent to this study is described below. This study was approved by the California State University, Fresno Committee on Protection of Human Subjects.

The participants eligible for the fall prevention screening had to meet all of the following

\section{Inclusion Criteria}

a) Have self-reported balance deficit that was limiting their functional activities, or have a history and/or fear of falls;

b) Be able to get out of a chair ind ependently;

c) Ambulate at least 50 feet without the use of an assistive device;

d) Receive physician clearance to participate.

\section{Exclusion criteria}

a) Diagnosed with dementia, Alzheimer's disease;

b) Have cardiac condition that can limit participation in physical activity (i.e., exercise); and

c) Extreme hypertension at or above 220/110mmh.

A total of 561 participants were included in the database. However, for the purpose of this study, only first time attendees who were community-dwelling adults
65 years or older were included. The final analysis sample consisted of 509 participants.

Data collected for all fall prevention screenings were completed by students who were trained as evaluators from the Department of Physical Therapy and School of Nursing at California State University, Fresno. These students were under the direct supervision of a licensed physical therapist and advanced practice nurse. Data collected during each screening were entered into a general spreadsheet using SPSS version 21. To maintain patient privacy, an identification number was assigned to each participant to replace his or her name in the databank. The information collected during the subjective portion of a screening included general sociodemographics (i.e., gender, age, educational level, living alone); health/activity questionnaire for self-reported physical activity level and past medication history where co morbidities were classified under cardiovascular (i.e., hypertension, congestive heart failure, peripheral vascular disease); neurological (i.e., stroke, dizziness, inner ear problem, numbness/tingling in lower extremity), endocrine (i.e., diabetes mellitus), osteoporosis, arthritis, or other.

Only medications consumed by participants in the last two weeks and prescribed by a physician were considered part of the medication inventory. To reduce underreporting, participants were asked to bring their medication lists and/or containers. Reason of indication was subjectively reported by the participant.

In order to collect data pertaining to the occurrence of falls, participants were asked, "In the last 12 months, have you experienced a fall?" The operational definition of a fall is an event which results in a person coming to rest inadvertently on the ground or floor or other lower level. For those answering in the affirmative, they were asked the following follow up questions: "How many times did you fall in the previous 12 months?", "Has any fall in the last 12 months resulted in a fracture and/or required medical treatment (i.e., hospitalization)?" Participants were identified as "fallers" if they have experienced two or more falls in the last 12 months. Individuals who reported no falls or only one fall served as the comparison group.

For this retrospective study, one person was responsible for identifying medications that belonged to the following categories: psychotropic, opioid, or cardiovascular. All medications were converted to their generic names. Medications were classified psychotropic if they crossed the blood brain barrier and primarily 


\section{Annals of Physiotherapy \& Occupational Therapy}

affected the central nervous system (CNS). Medications that bound to opioid receptors and were prescribed to relieve pain were classified under the opioid category. Lastly, classification into the cardiovascular category was any medication prescribed for diseases related to the structure and function of the heart and blood vessels identified under one of these subcategories: beta-blocker, angiotensin converting enzyme(ACE) inhibitor, angiotensin II receptor blocker (ARBs), vasodilator, calcium channel blocker (CCB), diuretic, anti-arrhythmic, alpha I blocker, cardiac glycoside, rennin inhibitor, or alpha II agonist [16]. Epocrates ${ }^{\circledR}$ App [31] Drugs.com $\bigodot$ [32] website were used as references for converting medications from brand names to generic names.

The frequency of use of medications identified as psychotropic, opioid, and cardiovascular was recorded in one of the three corresponding categories. This information was then re-coded, where one represented user of medication and zero represented non-user of medication. After data analysis was completed, it became apparent that further sub categorization of cardiovascular medications was required. Within the cardiovascular medication class, several pharmacological classes exist. To limit the potential for over generalization, all previously identified cardiovascular medications were recoded under one of the following cardiovascular subcategories based on mechanism of action: betablocker, ACE inhibitor, angiotensin II receptor blocker (ARBs), nitrate, vasodilator, calcium channel blocker, diuretic, anti-arrhythmic, alpha 1 blocker, cardiac glycoside, rennin inhibitor, alpha II agonist, and other for any cardiovascular medication that did not correspond to any category mentioned above.

Statistical analyses were completed using SPSS version 21. A frequency analysis was completed to obtain descriptive statistics for this sample. Since this research is exploratory in nature, a backward logistic regression analysis was conducted. Initially, the logistic regression analysis was implemented, with all predictors inserted into the model. It permitted the removal of individual predictor variables from the model (i.e., cardiovascular, psychotropic, opioids), taking into consideration the variables that were statistically significant. This procedure entered all predictor variables and next sequentially removed the non-significant variables in each step. The backward stepwise logistic regression model assisted in comparing which variable(s) predicted the greatest number of falls within the same sample. Based on these results, a possible interaction may have been occurring between two medication categories. Consequently, an interaction variable was created and a logistic regression model was calculated to predict number of falls in subjects who simultaneously consumed both psychotropic and opioid medications.

A series of chi-square $\left(\chi^{2}\right)$ tests of independence between dichotomously coded falls and the three major medication categories were conducted. Additional chisquare tests were performed for each cardiovascular medication subcategory due to the various types of medication classes within this category. The OR was then calculated to quantify the association between use of specific medication category and the reported incidence of falls as compared to non-users. Statistical significance was set at $\mathrm{P}<.05$ for all analyses.

\section{Results}

The mean age of participants was $77.7 \pm 7.2$ years. Participants were predominantly Caucasian (76\%), female $(79 \%)$, had at least a high school education level (74.6\%), and most reported living alone (58.2\%). The mean number of comorbidities reported per participant was $3.6 \pm 2.1$, with the majority reporting cardiovascular disease. Other prevalent health conditions included arthritis (57.8\%), neurological disease (51.9\%), and osteoporosis $(27.8 \%)$. Overall, most of the participants were physically active $(83.4 \%)$.

The mean number of reported falls was $1.5 \pm 3.4$. Based on the number of self-reported falls, a total of $68.8 \%$ of participants were classified as non-fallers $(\leq 1$ fall) and $31.2 \%$ of participants were identified as fallers ( $\geq 2$ falls). The mean number of prescribed medications was $5.2 \pm 3.31$. A total of 348 (67.8\%) participants reported consuming one or more cardiovascular medications, 123 (24\%) were consuming psychotropic medications, and $54(10.5 \%)$ participants reported consuming an opioid based analgesic. A total of 28 (5.5\%) individuals reported consuming both opioid and psychotropic medications. Table 1 contains the breakdown of the frequencies of usage of medications for each category: psychotropic, opioid, and cardiovascular. Table 2 contains a comparison between faller versus nonfaller and medication use for each category.

The findings of the logistic regression analysis with a backward stepwise condition are presented in Table 3 . The first logistic regression model included psychotropic, opioid, and cardiovascular as the independent variables. The three steps were then sequentially derived and each had only psychotropic as the significant variable. Each step eliminated a non-significant variable until step three, when only the psychotropic medication variable was left. 
However, the best model for prediction of fallers and nonfallers was in step two. In this step, psychotropic and opioid medications were in the model. This led to analyzing the combined effects or interaction between psychotropic and opioid categories as the variable, which resulted in a significant model with the best prediction and highest odds ratio. In this model, $70.3 \%$ of fallers and non-fallers were categorized correctly when patients were taking psychotropic and opioid medications. After calculating the interaction variable, it was concluded that the community-dwelling older adults 65 years and older who reported falls were taking psychotropic and opioid medication.

\begin{tabular}{|c|c|}
\hline Medications & n (\%) \\
\hline Psychotropic Medications & $123(24.0)$ \\
\hline Opioid Medications & $54(10.5)$ \\
\hline Cardiovascular Medications & $348(67.8)$ \\
\hline Beta Blocker & $165(32.2)$ \\
\hline ACE Inhibitor & $116(22.6)$ \\
\hline Angiotensin II Receptor Blocker & $91(17.7)$ \\
\hline Vasodilator & $36(7.0)$ \\
\hline Calcium Channel Blocker & $99(19.3)$ \\
\hline Diuretic & $144(28.1)$ \\
\hline Anti-arrhythmic & $15(2.9)$ \\
\hline Alpha I Blocker & $10(1.9)$ \\
\hline Cardiac Glycoside & $13(2.5)$ \\
\hline Renin Inhibitor & $3(0.6)$ \\
\hline Alpha II Agonist & $7(1.4)$ \\
\hline Other & $1(0.2)$ \\
\hline Use of 2 or more agents & $183(35.8)$ \\
\hline Psychotropic and Opioid & $28(5.5)$ \\
\hline Psychotropic and Cardiovascular & $94(18.4)$ \\
\hline Opioid and Cardiovascular & $41(8.0)$ \\
\hline Psychotropic, Opioid, and Cardiovascular & $20(3.9)$ \\
\hline
\end{tabular}

Table 1: Frequency of Use of Medication Categories.

\begin{tabular}{|c|c|c|c|}
\hline Medication Use & Psychotropic & Opioid & Cardiovascular \\
\hline Non-Faller n (\%) & $65(53.7)$ & $29(53.7)$ & $229(66.2)$ \\
\hline Faller n (\%) & $56(46.3)$ & $25(46.3)$ & $117(33.8)$ \\
\hline
\end{tabular}

Table 2: Non-faller vs. Faller and Medication Use (n=509).

\begin{tabular}{|c|c|c|c|c|c|c|c|c|c|c|}
\hline \multicolumn{11}{|c|}{ Logistic Regression Backward Stepwise $\mathbf{n = 5 0 9}$} \\
\hline Step 1 & Medications & B & S.E & Wald & df & P value & Predicted & Observed $\leq 1$ & Observed $\geq 2$ & $\%$ Correct \\
\hline & Cardiovascular & 0.3 & 0.22 & 1.92 & 1 & 0.16 & $\leq 1$ fall & 343 & 7 & 98 \\
\hline & Psychotropic & 0.76 & 0.22 & 11.77 & 1 & 0.001 & $\geq 2$ falls & 146 & 13 & 8.2 \\
\hline & Opioid & 0.48 & 0.19 & 43.88 & 1 & $<.001$ & Overall \% & & & 69.9 \\
\hline & Constant & 0.79 & 0.09 & 68.07 & 1 & $<.001$ & & & & \\
\hline \multirow[t]{4}{*}{ Step 2} & Medications & B & S.E & Wald & df & P value & Predicted & Observed $\leq 1$ & Observed $\geq 2$ & \% Correct \\
\hline & Psychotropic & 0.79 & 0.22 & 12.77 & 1 & $<.001$ & $\leq 1$ fall & 340 & 10 & 97.1 \\
\hline & Opioid & 0.49 & 0.3 & 2.59 & 1 & 0.11 & $\geq 2$ falls & 141 & 18 & 11.3 \\
\hline & Constant & 1.06 & 0.12 & 80.18 & 1 & $<.001$ & Overall \% & & & 70.3 \\
\hline \multirow[t]{2}{*}{ Step 3} & Medications & B & S.E & Wald & df & P value & Predicted & Observed $\leq 1$ & Observed $\geq 2$ & \% Correct \\
\hline & Psychotropic & 0.87 & 0.22 & 16.24 & 1 & $<.001$ & $\leq 1$ fall & 350 & 0 & 100 \\
\hline
\end{tabular}




\section{Annals of Physiotherapy \& Occupational Therapy}

\begin{tabular}{|c|c|c|c|c|c|c|c|c|c|c|c|}
\hline & Constant & 1.02 & 0.12 & 78.37 & 1 & 0.36 & $\geq 2$ falls & 159 & 0 & 0 \\
\hline \multicolumn{8}{|c|}{ Logistic Regression for Combination of Medications n=509 } \\
\hline \multicolumn{8}{|c|}{} & \multicolumn{8}{|c|}{ Step 1 } & Medications & B & S.E & Wald & df & P value & Predicted & Observed $\leq 1$ & Observed $\geq 2$ & \% Correct \\
\hline & $\begin{array}{c}\text { Psychotropic } \\
\text { and Opioid }\end{array}$ & 1.47 & 0.41 & 13.01 & 1 & $<.001$ & $\leq 1$ fall & 340 & 10 & 98 \\
\hline & Constant & 0.88 & 0.1 & 77.22 & 1 & $<.001$ & $\geq 2$ falls & 141 & 18 & 8.2 \\
\hline & & & & & & & Overall \% & & & 70.3 \\
\hline
\end{tabular}

Table 3: Logistic Regression Models.

A chi-square test of independence for all three medications by fall category was conducted. Chi-square test analysis detected a statistical significance between reported incidence of falls and use of the following medications: psychotropic $\left(\chi^{2}=16.72, \mathrm{P}<.001\right)$ and opioid $\left(\chi^{2}=6.38, \mathrm{P}<.001\right)$. Cardiovascular medications, when analyzed as a single category, were not statistically significant $\left(\chi^{2}=3.341, P=.06\right)$. For cardiovascular medication subcategories, only one subcategory was statistically significant anti-arrhythmic medication users $\left(\chi^{2}=12.75, \mathrm{P}<.001\right)$. Only 15 participants $(2.9 \%)$ reported use of anti-arrhythmic agents, however, most of these participants $(n=11,73.3 \%)$ reported falls, while four participants $(26.7 \%)$ were classified as non-fallers. All other cardiovascular subcategories were not statistically significant Table 4.

\begin{tabular}{|c|c|c|c|}
\hline Pearson Chi Square & Value & df & P value \\
\hline Psychotropic & 16.72 & 1 & $<.001$ \\
\hline Opioid & 6.38 & 1 & 0.01 \\
\hline Cardiovascular & 3.34 & 1 & 0.06 \\
\hline Anti-arrhythmic & 12.75 & 1 & $<.001$ \\
\hline
\end{tabular}

Abbreviation: df, degrees of freedom

Table 4: Chi-Square Test Results.

Based on the self-reported falls, the calculated odds of experiencing a fall are reported in Table 5. Participants who consumed psychotropic only, opioids only, or cardiovascular medications only had an OR of 2.38 (95\% CI 1.56-3.64), 2.07 (95\% CI 1.17-3.66), and 1.47 (95\% CI, 0.97-2.23), respectively. However, for participants who consumed both psychotropic and opioid medications, the reported OR was 4.3 (95\% CI 1.96-9.64).

\begin{tabular}{|c|c|c|}
\hline Medications & OR & $\mathbf{( 9 5 \% ~ C I ) ~}$ \\
\hline Psychotropic & 2.4 & $1.56-3.64$ \\
\hline Opioid & 2.1 & $1.17-3.66$ \\
\hline Cardiovascular & 1.5 & $0.97-2.23$ \\
\hline Psychotropic and Opioid & 4.3 & $1.96-9.64$ \\
\hline
\end{tabular}

Table 5: Odds Ratio (OR).

\section{Discussion}

This retrospective study demonstrated an association between fall incidence and the use of psychotropic and opioid medications. Among the subcategories within the cardiovascular medication class, only the use of antiarrhythmic medication was reported to have an association with fall incidence. A statistically significant model for predicting faller and non-faller incidence was found. The best predictor of falls $(70.3 \%$ of the time) was the concurrent use of psychotropic and opioid medications. The odds of experiencing a fall doubles (4.3 times more likely to experience a fall) when subjects reported both medications as opposed to psychotropic or opioid use alone (2.4 and 2.1 times greater respectively). This is possibly due to the increased sedating effect the use of both of these medications can create. Although dosages were not collected from participants, future research should examine this topic further. Hanlon, et al. [33] reported a three-fold increase in experiencing recurrent falls for individuals taking higher doses, as compared to those taking moderate doses of central nervous system medications.

The findings for psychotropic category, are consistent with other studies $[13,14,18,20]$. Leipzig, et al. [18] reported that individuals who used psychotropic medications were associated with the incidence of falls. The results from their meta-analysis have been replicated throughout the literature. Bloch, et al. [21] reported an association between fall incidence and the use of psychotropic medications when analyzed as a single medication class or by medication sub-categories. The data gathered from the aforementioned meta-analyses incorporated a wide variety of settings (i.e., long term care facilities, acute care hospital, and community). Although several of these meta-analyses varied in their methodology, these studies reported an association between use of psychotropic medications and fall incidence. This same association has also been reported in individual studies that included only community-dwelling adults aged 65 years and older [20,22]. Payne, et al. [30] 
and Vitry, et al. [34] included both genders in their sample, but had predominantly more females than males who reported use of psychotropic medication. Vitry, et al. [34] reported a higher risk of falls in females than in males who used psychotropic medications. Similar results were found in this investigation. The majority of participants in this investigation who reported the use of psychotropic medications were females $(n=101)$, of these participants $49.5 \%$ were identified as fallers. An association was detected between female participants who used psychotropic medications and fall incidence $\left(\chi^{2}\right.$ $=18.64, \mathrm{P}<.001)$. Vitry, et al. [34] suggested a possible explanation: an association between falls among females who used psychotropic medication and a greater susceptibility to psychomotor impairments (i.e., dizziness).

Furthermore, participants who used opioids as part of their medication regimen were associated with the incidence of falls. Of the participants in this study who reported use of opioid medication, $46.3 \%$ were classified as fallers. This is of concern due to the increased trend in use of opioid medication in older adults [23]. Similar to the psychotropic medication category, an observation was noted in which the majority of opioid users in this investigation were female $(n=24)$. From this group, the majority were classified as fallers (49\%). In this investigation, an association was detected between opioid use and fall incidence in the female gender $\left(\chi^{2}=7.48, P=\right.$ .01 ). Studies have reported an increased risk of injury with use of opioid medication associated with the female gender [35-37]. In a study, it was observed that individuals using opioid medications had a reported decrease in health and poorer functional level [37]. Other studies have attributed the use of opioid medications to the prevalence of certain co morbidities, such as osteoarthritis, which requires consistent pain management [25,27]. In addition, Rolita, et al. [25] found that older females with osteoarthritis, who consumed opioid medication for the management of pain, had an increased risk for injury, with a reported OR of 3.3 compared to users of COX-2 inhibitors for pain relief. In this investigation, similar demographics were reported, where $61.8 \%$ of the sample reported having been medically diagnosed with some form of arthritis. A reported OR of 2.3 (95\% CI 1.3-4.2) of experiencing a fall was noted for the female sample. The difference between the results of this investigation and the results reported by Rolita, et al. [25] may be due to the small sample and reported number of falls. Nevertheless, the findings in this investigation substantiate that female opioid users are likely to experience falls.
Although cardiovascular medications are the most commonly used medications in older adults, no association was found between their use and fall incidence, with the exception of anti-arrhythmic medication. Regarding anti-arrhythmic medication, this finding was consistent with the results of previous studies [28,38]. According to Hartikainen, et al. [5] sub categorization of cardiovascular medication is a recommended step due to the wide variety of medications that act differently on specific receptors. The majority, if not all, studies that accessed cardiovascular medication and fall incidence incorporated this methodology $[14,28,30]$. In this investigation, although the sample size decreased with sub categorization of the cardiovascular medications, the association between medication subcategories and falls became more apparent. Although only $2.9 \%$ of community-dwelling older adults reported use of anti-arrhythmic medication in this study, a reported fall incidence of $73.3 \%$ was noted. This association was also observed when assessed independently based on gender. Both males $\left(\chi^{2}=10.32\right.$, $\mathrm{P}=.01)$ and females $\left(\chi^{2}=5.24, \mathrm{P}=.02\right)$ were found to be independently associated with fall incidence and the use of anti-arrhythmic medications. This investigation captured a small snapshot of the association between fall incidence and use of anti-arrhythmic medication. However, in this investigation the association between anti-arrhythmic medication and fall incidence may have been overestimated due to the small sample allocated to this medication subcategory.

One of the identified mechanisms related to the occurrence of falls and use of cardiovascular medications, such as anti-arrhythmic, related to the potential occurrence of a hypotensive event $[29,38]$. This study used data collected from the fall prevention screenings, which required participants to retrieve physician clearance prior to participation and screened for orthostatic hypotension. Therefore, it can be speculated that the sample for this study was less likely to experience a hypotensive event. Further studies are needed in assessing if a true association exists between use of cardiovascular medications and fall incidence.

This study has a number of strengths. It captures an association between fall incidence and the use of two of three most frequently prescribed medication categories. The classification of medication into their respective categories, based on the mechanism of action, provides a more general screen. Also, a more standardized operational fall definition was implemented that has been recognized globally. However, there are several limitations to this study worth reviewing. As a 
retrospective study, no true statement of causation can be made. There is a potential for confounding effect due to comorbidities, health status, and age. In addition, participants subjectively reported only list of medications consumed in the last two weeks, potentially limiting the reporting of medications or capturing use of some short term medications. Furthermore, some medications may take longer than two weeks to achieve the full dosing effect, overestimating their contribution. Although the findings of this study identified an association among the use of psychotropic, opioid, or both medications, inclusion of medication dosage and duration of use is still needed.

\section{Conclusion}

The findings of this study reinforce the concept that individuals, who consume a type of psychotropic, opioid, or both, are associated with fall incidence. Subjects in this study were 4.3 times more likely to report a fall when taking medication from both classifications as opposed to only one. Thus, it is important for clinicians like physical therapists, to identify patients who are using these medications. As the number of older adults seeking physical therapy services increases, physical therapists particularly in direct access states must be proactive with medication reviews including psychotropic or opioid medications to promote reduced fall incidence within this population group. In an attempt to lower forthcoming medical expenditures, health case management will continue to promote preventative measures. This change in the healthcare model amplifies the importance of proactively screening patient's medication.

\section{Acknowledgements}

Funding Support: This study was supported by The California Wellness Foundation

Role of the Sponsor: The funding provided had no role in the design and conduct of the study, management, analysis of the data, or preparation, review, or approval of the manuscript.

\section{References}

1. Werner C (2011) The older population: 2010. Census Briefs 2011.

2. Tromp AM, Pluijm SM, Smit JH, Deeg DJ, Bouter LM, et al. (2001) Fall-risk screening test: A prospective study on predictors for falls in community-dwelling elderly. J Clin Epidemiol 54(8): 837-844.
3. Berry SD, Miller R (2008) Falls: Epidemiology pathophysiology and relationship to fracture. Curr Osteoporos Rep 6(4): 149-154.

4. Freeland KN, Thompson AN, Zhao Y, Leal JE, Mauldin PD, et al. (2012) Medication use and associated risk of falling in a geriatric outpatient population. Ann Pharmacother 46(9): 1188-1192.

5. Hartikainen S, Lonnroos E, Louhivuori K (2007) Medication as a risk factor for falls: Critical systematic review. J Gerontol A Biol Sci Med Sci 62(10): 11721181.

6. Volkow N (2014) Prescription drug abuse.U.S. Department of Health and Human Services, National Institutes of Health, National Institute on Drug Abuse.

7. Hajjar ER, Cafiero AC, Hanlon JT (2007) Polypharmacy in elderly patients. Am J Geriatr Pharmacother 5(4): 345-351.

8. Maher RL, Hanlon J, Hajjar ER (2014) Clinical consequences of polypharmacy in elderly. Expert Opin Drug Saf 13(1): 57-65.

9. Kojima T, Akishita M, Nakamura T (2011) Association of polypharmacy with fall risk among geriatric outpatients. Geriatr Gerontol Int 11 (4): 438-444.

10. Kelly K, Pickett W, Yiannakoulias N (2003) Medication use and falls in community-dwelling older persons. Age Ageing 32: 503-509.

11. Soni A, Roemer M (2013) Expenditures for the top five therapeutic classes of outpatient prescription drugs, medicare beneficiaries, age 65 and older, U. S. civilian noninstitutionalized population 2010. Statistical Brief \# 410. Agency for Healthcare Research and Quality, Rockville, MD.

12. Berdot S, Bertrand M, Dartigues JF (2009) Inappropriate medication use and risk of falls-A prospective study in a large community-dwelling elderly cohort. BMC Geriatr 9:30.

13. Woolcott JC, Richardson KJ, Wiens MO (2009) Metaanalysis of the impact of 9 medication classes on falls in elderly persons. Arch Intern Med 169(21): 19521960.

14. Cumming RG (1998) Epidemiology of medicationrelated falls and fractures in the elderly. Drug Aging 12(1): 43-53.

15. Hill KD, Wee R (2012) Psychotropic drug-induced falls in older people. Drug Aging 29(1): 15-30. 
16. Panus $P$, Katzung B, Jobst E, Tinsley S, Masters S et al. (2008) Pharmacology for the Physical Therapist. $1^{\text {st }}$ (Edn.), McGraw Hill, New York, USA, pp: 30-133.

17. Chrischilles EA, VanGilder R, Wright K, Kelly M, Wallace RB (2009) Inappropriate medication use as a risk factor for self-reported adverse drug effects in older adults. J Geriatr Soc 57(6): 1000-1006.

18. Leipzig R, Cumming R, Tinetti M (1999) Drugs and falls in older people: A systematic review and metaanalysis: I. Psychotropic drugs. J Am Geriatr Soc 47(1): 30-39.

19. van Strien AM, Koek HL, van Marum RJ, EmmelotVonk MH (2013) Psychotropic medications, including short acting benzodiazepines, strongly increase the frequency of falls in elderly. Maturitas 74(4): 357362.

20. Chaimowicz F, Ferreira Tde J, Miguel DF (2000) Use of psychoactive drugs and related falls among older people living in a community in Brazil. Rev Saude Publica 34(6): 631-635.

21. Bloch F, Thibaud M, Dugue B, Breque C, Rigaud AS (2011) Psychotropic drugs and falls in the elderly people: Updated literature review and meta-analysis. J Aging Health 23(2): 329-346.

22. Weiner DK, Hanlon JT, Studenski SA (1998) Effects of central nervous system polypharmacy on falls liability in community-dwelling elderly. Gerontology 44(4): 217-221.

23. Sawyer P, Bodner EV, Ritchie CS, Allman RM (2006) Pain and pain medication use in community-dwelling older adults. Am J Geriatr Pharmacother 4(4): 316324.

24. Chau DL, Walker V, Pai L, Cho LM (2008) Opiates and elderly: Use and side effects. Clin Interv Aging 3(2): 273-278.

25. Rolita L, Spegman A, Tang X, Cronstein BN (2013) Increasing narcotic analgesic prescriptions for osteoarthritis is associated with increased falls and fractures in the elderly. J Am Geriatr Soc 61(3): 335340 .

26. Leipzig RM, Cumming RG, Tinetti ME (1999) Drugs and falls in older people: A systematic review and meta-analysis: II. Cardiac and analgesic drugs. J Am Geriatr Soc 47(1): 40-50.
27. Buckeridge D, Huang A, Hanley J (2010) Risk of injury associated with opioid use in older adults. J Am Geriatr Soc 58(9): 1664-1670.

28. Darowski A, Whiting R (2011) Cardiovascular medication and falls. Rev Clin Gerontol 21(2): 170179.

29. Zang G (2013) Antihypertensive drugs and the risk of fall injuries: A systematic review and meta-analysis. J Int Med Res 41(5): 1408-1417.

30. Payne R, Abel G, Simpson C, Maxwell S (2013) Association between prescribing of cardiovascular and psychotropic medications and hospital admission for falls or fractures. Drug Aging 30(4): 247-254.

31. Epocrates (2013).

32. Drugs.com (2014).

33. Hanlon JT, Boudreau RM, Roumani YF (2009) Number and dosage of central nervous system medications on recurrent falls in community elders: The health, aging and body composition study. J Gerontol A Biol Sci Med Sci 64(4): 492-498.

34. Vitry AI, Hoile AP, Gilbert AL, Esterman A, Luszcz MA (2010) The risk of falls and fractures associated with persistent use of psychotropic medications in elderly people. Arch Gerontol Geriatr 50(3): 1-4.

35. Campbell CI, Weisner C, Leresche L (2010) Age and gender trends in long-term opioid analgesic use for noncancer pain. Am J Public Health 100(12): 25412547.

36. Saunders KW, Dunn KM, Merrill JO (2010) Relationship of opioid use and dosage levels to fractures in older chronic pain patients. J Gen Intern Med 25(4): 310-315.

37. Rogers KD, Kemp A, McLachlan AJ, Blyth F (2013) Adverse selection? A multi-dimensional profile of people dispensed opioid analgesics for persistent non-cancer pain. PLoS One 8(12): e80095.

38. Lee HC, Tl Huang K, Shen WK (2011) Use of antiarrhythmic drugs in elderly patients. J Geriatr Cardiol 8(3): 184-194. 